\title{
Non-contact Intraocular Pressure Measurement over Bandage Contact Lens and the Effect of Pentacam and Corvis ST's IOP Correction System
}

\author{
Xueting Cai \\ Wenzhou Medical University \\ Yi Qin \\ Wenzhou Medical University \\ Sixiu Liu \\ Wenzhou Medical University \\ Zhewei Cheng \\ Wenzhou Medical University \\ Fan Lu \\ Wenzhou Medical University \\ Jia Qu \\ Wenzhou Medical University \\ Ding Chen ( $\nabla$ necoding@126.com ) \\ Wenzhou Medical University
}

\section{Research Article}

Keywords: bandage contact lens (BCL), non-contact tonometer (NCT), Pentacam, Corvis ST, IOP correction

Posted Date: March 1st, 2022

DOI: https://doi.org/10.21203/rs.3.rs-1342670/v1

License: (c) (1) This work is licensed under a Creative Commons Attribution 4.0 International License. Read Full License 


\section{Abstract}

Purpose: To evaluate the influence of intraocular pressure (IOP) measurement while wearing bandage contact lens (BCL) and the effect of Pentacam and Corvis ST's correction systems.

Methods: It was a prospective comparative study. Forty healthy subjects (Forty eyes) were included in this study. Eyes were measured by non-contact tonometer (NCT), Corvis ST and Pentacam before and after wearing BCL. Pentacam's five correction formulas (Ehlers formula, Shah formula, Dresden formula, Kohlhaas formula, Orssengo/Pye formula) and Corvis ST's correction formulas (Ehlers formula and biomechanical corrected formula) were used to correct the IOP values before and after BCL wearing. The IOP values were compared and the correction effect of different systems were evaluated.

Results: The mean age of the subjects was $24.4 \pm 0.60$ years. The mean IOP obtained by NCT was $14.8 \pm 3.2 \mathrm{mmHg}$ before $\mathrm{BCL}$ wearing, and was $15.7 \pm 3.4 \mathrm{mmHg}$ after $\mathrm{BCL}$ wearing. The mean IOP was significantly increased after $B C L$ wearing $(0.9 \pm 2.9 \mathrm{mmHg}, P=0.05)$. Four of the five Pentacam's correction formulas (except Kohlhaas formula) showed no significant difference in the mean corrected IOP values before and after BCL wearing (all P>0.05). The mean IOP obtained by Corvis ST was $13.7 \pm 2.8 \mathrm{mmHg}$ before $B C L$ wearing, and was $15.0 \pm 4.0 \mathrm{mmHg}$ after $B C L$ wearing. The mean IOP was significantly increased after BCL wearing $(1.3 \pm 2.4 \mathrm{mmHg}, \mathrm{P}<0.05)$. Corvis ST's correction formula (Ehlers formula other than biomechanical corrected formula) showed no significant difference in the mean corrected IOP values before and after $B C L$ wearing $(P>0.05)$.

Conclusion: The IOP measurements over BCL by NCT and Corvis ST was found to be increased. The correction systems of Pentacam (Ehlers formula, Shah formula, Dresden formula, and Orssengo/Pye formula) and Corvis ST (Ehlers formula) are useful in correcting the IOP measuring deviation induced by $\mathrm{BCL}$ wearing.

\section{Introduction}

Bandage contact lens $(\mathrm{BCL})$ is an effective treatment method for persistent epithelial defects, recurrent corneal erosions, filamentous keratitis, corneal surface irregularities, corneal abrasions, corneal thinning, bullous keratopathy, thermal and chemical burns, and ocular surface reconstruction ${ }^{1-4}$. Intraocular pressure (IOP) is a fundamental and essential ocular parameter in ophthalmological clinics. Frequent measurement of IOP may be needed in some patients during the follow-up. Measuring IOP over BCL is convenient, however, $\mathrm{BCL}$ wearing may affect the results of IOP measurement. The frequent removal of $B C L$ for the purpose of accurate IOP measurement may negatively affect corneal epithelization and delay the recovery process. Therefore, correction of IOP to obtain the accurate values is important for BCL wearers in cases where contact lens removal is not desired.

Pentacam is an anterior segment imaging and analyzing system. It has five built-in IOP correction formulas (Ehlers, Shah, Dresden, Kohlhaas, and Orssengo/Pye), which can correct the effects of corneal thickness and corneal curvature of the input IOP values from other tonometers ${ }^{5}$. BCL wearing will 
increase the apparent corneal thickness measured by Pentacam, however, using Pentacam's built-in IOP correction system to calibrate the IOP values with BCL has not been previously reported. Corvis ST is a new type of non-contact tonometer. It can provide IOP values, corneal thickness and biomechanical parameters ${ }^{6}$. It can also provide corrected IOP values which is claimed to be less affected by cornea's stiffness ${ }^{7,8}$. BCL wearing will change the biomechanics of ocular surface, and using Corvis ST to correct the IOP values with BCL has not been previously reported.

In this study, we investigated the influence of silicone hydrogel BCL wearing on the IOP measurement using different tonometries (NCT and Corvis ST) and evaluate the effect of calibration by Pentacam and Corvis ST's IOP correction systems respectively.

\section{Methods}

\section{Subjects and materials}

This was a prospective comparative study. A total 40 eyes of 40 volunteers ( 22 males, 18 females) who did not have any ocular or systemic disease were included in the study from January 2019 to December 2019 in the Eye Hospital of Wenzhou Medical University. Institutional Review Board approval were obtained from the Human Research Ethics Committee at Wenzhou Medical University. Informed consent was obtained from all subjects. The study was conducted under the ethical standards outlined in the Declaration of Helsinki.

The inclusion criterion was a normal cornea confirmed with no degeneration or dystrophy after a full ophthalmologic examination. The exclusion criteria were any systemic or ocular disease or any history of ocular surgery. Subjects with corneal astigmatism higher than $2.00 \mathrm{D}$, and those using soft contact lens in recent 2 weeks or using rigid contact lens in recent 1 month, and those having a history of contact lens intolerance were also excluded.

The contact lenses used in this study were silicone hydrogel lenses (Sure sight, Alcon, Fort Worth, TX, USA) with $24 \%$ water content and $0 \mathrm{D}$ power. The base curvature and the diameter of the lenses were 8.60 $\mathrm{mm}$ and $13.80 \mathrm{~mm}$ respectively.

\section{Measurements}

A full ophthalmic examination was performed on each subject, including visual acuity measurement and slit lamp biomicroscopy for anterior and posterior segment evaluation with a $90 \mathrm{D}$ lens. The central corneal thickness (CCT) was measured with Pentacam Scheimpflug imaging system (Oculus Optikgeräte $\mathrm{GmbH}$, Wetzlar, Germany). The instruments used for IOP measurements were a non-contact tonometer (NCT, Topcon CT-80A Computerized Tonometer; Topcon, Tokyo, Japan) and Corvis ST (Oculus Optikgerate $\mathrm{GmbH}$, Wetzlar, Germany). Pentacam's IOP correction system and Corvis ST's IOP correction system were applied to correct IOP values obtained. The IOP correction formulas in Pentacam's and Corvis ST's IOP correction systems are listed as follows. 
1. Ehlers formula ${ }^{9} \rrbracket \Delta \mathrm{IOP}=0.071 \times(545 \mu \mathrm{m}-\mathrm{CCT})$

2. Shah formula ${ }^{10} \nabla \Delta \mathrm{IOP}=0.050 \times(550 \mu \mathrm{m}-\mathrm{CCT})$

3. Resden formula ${ }^{11} \rrbracket \Delta \mathrm{IOP}=0.040 \times(550 \mu \mathrm{m}-\mathrm{CCT})$

4. Kohlhaas formula ${ }^{12} \rrbracket \mid \mathrm{OP}=\mathrm{IOP}($ measured $)+(540-\mathrm{CCT}) / 71+(43-\mathrm{K}) / 2.7+0.75 \mathrm{mmHg} \rrbracket(\mathrm{K} \rrbracket$ corneal curvature $)$

5. Orssengo/Pye formula ${ }^{13} \bowtie \mathrm{IOP}=\mathrm{IOP}($ measured $) / \mathrm{K} \otimes \mathrm{K}=(\mathrm{Bc}-\mathrm{C} \mathrm{C}+\mathrm{C}) / \mathrm{B}$

6. $\mathrm{BIOP}^{7,14}=\left(\mathrm{C}_{\mathrm{CCT} 1} \times \mathrm{C}_{\mathrm{CVSIOP}}+\mathrm{C}_{\mathrm{CCT} 2}\right) \times \mathrm{C}_{\mathrm{Age}}$

$\mathrm{C}_{\mathrm{CCT} 1}=4.67 \times 10^{-7} \times \mathrm{CCT}^{2}-7.8 \times 10^{-4} \times \mathrm{CCT}+0.63$

$\mathrm{C}_{\mathrm{CCT} 2}=-1.73 \times 10^{-5} \times \mathrm{CCT}^{2}+2.02 \times 10^{-3} \times \mathrm{CCT}-0.97$

$\mathrm{C}_{\mathrm{CVSIOP}}=10+(\mathrm{CVS}-\mathrm{IOP}+1.16) / 0.389$

$\mathrm{C}_{\text {Age }}=-2.01 \times 10^{-5} \times \mathrm{Age}^{2}+1.3 \times 10^{-3} \times$ Age +1

BIOP: biomechanical corrected IOP values by Corvis ST

\section{Procedures}

Three different measurements were taken on the right eye of each subject. The first IOP measurement of the naked eyes was implemented by NCT, three consecutive measurement values were made, and the average IOP values were recorded. Then all of the subjects were examined with Pentacam. Five IOP correction formulas of Pentacam would provide corresponding corrected IOP values. Also, the naked eyes were implemented by Corvis ST, the IOP and corrected IOP were recorded. After those procedures finished, subjects wore BCLs for 10 minutes, and were then measured with these three instruments again. All data were recorded. All of the examinations were carried out between 8:00 am to 10:00 am.

\section{Statistical analysis}

Statistical analysis was performed by Statistical Package for Social Science (SPSS 22.0 for Windows). Paired t-test was used to analyze the difference between the IOP values with and without BCLs. The difference between the IOP values of naked eyes' and the corrected IOP values of eye with BCLs was also analyzed. Bland-Altman plots was used to assess the agreement between the IOP values from NCT and Corvis ST. For the Bland-Altman plots, $95 \%$ limits of agreement were set as acceptable values. $\mathrm{P}<0.05$ value was accepted as statistically significant.

\section{Results}


The mean IOP obtained by NCT was $14.8 \pm 3.2 \mathrm{mmHg}$ before BCL wearing, and was $15.7 \pm 3.4 \mathrm{mmHg}$ after BCL wearing. The mean IOP value was significantly higher after $B C L$ wearing $(0.9 \pm 2.9 \mathrm{mmHg}, \mathrm{P}=$ 0.04). The mean IOP obtained by Corvis ST was $13.7 \pm 2.8 \mathrm{mmHg}$ before $B C L$ wearing, and was $15.0 \pm$ $4.0 \mathrm{mmHg}$ after $\mathrm{BCL}$ wearing. The mean IOP value was significantly higher after $\mathrm{BCL}$ wearing $(1.3 \pm$ $2.4 \mathrm{mmHg}, \mathrm{P}=0.002$ ) (Table 1). The Bland-Altman plots for the comparison between the IOP values obtained by NCT and Corvis ST before and after BCL wearing are shown in Fig. 1 and Fig. 2 respectively. Both figures showed the IOP values by NCT was higher than Corvis ST before and after BCL wearing (+ $1.1 \mathrm{mmHg}$ and $+0.7 \mathrm{mmHg}$, respectively, both $\mathrm{P}<0.05$ ).

The mean native CCT before BCL wearing was $543 \pm 43 \mu \mathrm{m}$ measured by Pentacam. After BCL wearing, the apparent CCT including the thickness of cornea, BCL and tear film in between was $555 \pm 47 \mu \mathrm{m}$. The difference between the native CCT and the apparent CCT was statistically different $(P=0.01)$.

Pentacam's five correction formulas (Ehlers, Shah, Dresden, Kohlhaas, and Orssengo/Pye) were used to correct the IOP values obtained by NCT. There was no significant difference between the corrected values of IOP measured before and after BCL wearing (all $\mathrm{P}>0.05$ ) (Table 1). We also compared the difference between the IOP values obtained by NCT before BCL wearing and the corrected IOP values after BCL wearing by Pentacam's five correction formulas. Only IOP values corrected by formula Kohlhaas's showed significant difference $(1.4 \pm 2.9 \mathrm{mmHg}, \mathrm{P}=0.004)$, while the other four formulas (Ehlers, Shah, Dresden, and Orssengo/Pye) showed no significant difference (all $P>0.05$ ) (Table 2).

The Corvis ST IOP values were corrected by Ehlers formula and biomechanical formula. The corrected IOP values showed no significant difference before and after $B C L$ wearing (both $P>0.05$ ) (Table 1). The difference between the IOP values obtained by the Corvis ST before BCL wearing and the corrected IOP values after BCL wearing were also compared. The biomechanically corrected IOP (BIOP) values showed significantly difference $(+1.7 \pm 2.1 \mathrm{mmHg}, \mathrm{P}<0.001)$, while Corvis ST IOP values corrected by formula Ehlers showed no significant difference $(P=0.179)$ (Table 2).

Table1. IOP values Before and after BCL wearing $(n=40)$ 


\begin{tabular}{|lllll|}
\hline IOP(mmHg) & Before BCL wearing & After BCL wearing & D-value & P \\
\hline IOPt & $14.8 \pm 3.2$ & $15.7 \pm 3.4$ & $-0.9 \pm 2.9$ & 0.04 \\
\hline IOPC & $13.7 \pm 2.8$ & $15.0 \pm 4.0$ & $-1.3 \pm 2.4$ & 0.002 \\
\hline IOPe & $15.0 \pm 3.1$ & $15.0 \pm 3.3$ & $0.1 \pm 2.7$ & 0.87 \\
\hline IOPs & $15.2 \pm 2.8$ & $15.5 \pm 2.9$ & $-0.3 \pm 2.7$ & 0.464 \\
\hline IOPk & $15.1 \pm 2.8$ & $15.5 \pm 2.9$ & $-0.4 \pm 2.7$ & 0.318 \\
\hline IOPO & $15.4 \pm 3.0$ & $16.2 \pm 3.1$ & $-0.8 \pm 2.8$ & 0.072 \\
\hline IOPce & $15.1 \pm 3.0$ & $15.3 \pm 2.9$ & $-0.2 \pm 3.0$ & 0.624 \\
\hline BIOP & $11.6 \pm 2.0$ & $13.0 \pm 3.1$ & $0.5 \pm 2.5$ & 0.25 \\
\hline
\end{tabular}

BCL: bandage contact lens, NCT: non-contact tonometer, IOPt: NCT IOP values, IOPe: NCT IOP values corrected by formula Ehlers, IOPs: NCT IOP values corrected by formula Shah, IOPd: NCT IOP values corrected by formula Dresden, IOPk: NCT IOP values corrected by formula Kohlhaas, IOPo: NCT IOP values corrected by formula Orssengo/Pye, IOPc: Corvis ST IOP values, IOPce: Corvis ST IOP values corrected by formula Ehlers, BIOP: biomechanical corrected IOP values by Corvis ST.

Table2. Before (NCT) and after BCL wearing Paired Samples Test $(n=40)$

\begin{tabular}{|lll|}
\hline & D-value(mmHg) & $P$ \\
\hline IOPt1 - IOPe2 & $-0.2 \pm 4.1$ & 0.736 \\
\hline IOPt1 - IOPs2 & $-0.7 \pm 3.5$ & 0.212 \\
\hline IOPt1 - IOPd2 & $-0.7 \pm 3.2$ & 0.166 \\
\hline IOPt1 - IOPk2 & $-1.4 \pm 2.9$ & 0.004 \\
\hline IOPt1 - IOPo2 & $-0.5 \pm 3.3$ & 0.342 \\
\hline IOPc1-IOPce2 & $+0.7 \pm 3.2$ & 0.179 \\
\hline IOPc1-BIOP2 & $+1.7 \pm 2.1$ & $\leq 0.001$ \\
\hline
\end{tabular}

BCL: bandage contact lens, NCT: non-contact tonometer, IOPt1: NCT IOP values before BCL wearing, IOPt2: NCT IOP values after BCL wearing, IOPe2: NCT IOP values after BCL wearing corrected by formula Ehlers, IOPs2: NCT IOP values after BCL wearing corrected by formula Shah, IOPd2: NCT IOP values after $\mathrm{BCL}$ wearing corrected by formula Dresden, IOPk2: NCT IOP values after BCL wearing corrected by formula Kohlhaas, IOPo2: NCT IOP values after BCL wearing corrected by formula Orssengo/Pye, IOPc1: Corvis ST IOP values before BCL wearing, IOPc2: Corvis ST IOP values after BCL wearing, IOPce2: Corvis 
ST IOP values after BCL wearing corrected by formula Ehlers, BIOP2:biomechanical corrected Corvis ST IOP values after $B C L$ wearing.

\section{Discussion}

The IOP measurement was overestimated after bandage contact lens $(\mathrm{BCL})$ wearing, either by NCT $(0.9 \pm$ $2.9 \mathrm{mmHg})$ or by Corvis ST $(1.3 \pm 2.4 \mathrm{mmHg})$ in this study. This is consistent with previous studies ${ }^{15-18}$, while the average difference of IOP before and after contact lens wearing varies among these studies. Previous studies suggest that IOP measurement overestimation with soft contact lenses may be associated with lens power, central thickness, as well as the rigidity of the lens material ${ }^{16,19,20}$. BCL as a special type of soft contact lens, may affect the IOP measurement by those factors mentioned above. $\mathrm{BCL}$ wearing not only increases of the thickness of the eye wall that is flattened by the air puff of tonometer, but also changes the biomechanics of the cornea-BCL complexus.

Previous studies have confirmed that IOP measurement increases when central corneal thickness (CCT) increases. According to Zhang et al. ${ }^{21}$, the IOP measured by Goldmann applanation tonometer and NCT increased $0.39 \mathrm{mmHg}$ and $0.64 \mathrm{mmHg}$ respectively for each $10 \mu \mathrm{m}$ increase of CCT. Doughty et al. ${ }^{22}$ considered that the IOP difference was $3.4 \mathrm{mmHg}$ for every $10 \%$ difference in CCT. However, previous studies had different views on the effect of central thickness of soft contact lens on IOP measurement. Ogbuehi et al. ${ }^{20}$ considered that the effect of central thickness of soft contact lens with high water content was similar to the effect of central corneal thickness. They also deemed that the influence of high water content lenses with central thickness less than $0.3 \mathrm{~mm}$ could be ignored. Nevertheless, McMonnies et al. ${ }^{15}$ believed that the influence was significant and nonnegligible when the central thickness of the contact lens was greater than $0.15 \mathrm{~mm}$.

The wearing of $\mathrm{BCL}$ increased the apparent $\mathrm{CCT}$ measuring values by Pentacam or Corvis ST, which may partially explain the change of IOP measurement values. After BCL wearing, Pentacam indicated that the apparent CCT increased by $12 \pm 13 \mu \mathrm{m}$; according to Zhang et al. ${ }^{21}$, the corresponding increased IOP was about $0.768 \mathrm{mmHg}$; according to Doughty et al. ${ }^{22}$, the corresponding increased IOP was about 0.748 $\mathrm{mmHg}$; in our study, the measured IOP values by NCT increased $0.9 \pm 2.9 \mathrm{mmHg}$. After BCL wearing, Corvis ST indicated that apparent CCT increased by $24 \pm 15 \mu \mathrm{m}$; according to Zhang et al. ${ }^{21}$, the corresponding increased IOP was about $1.536 \mathrm{mmHg}$; according to Doughty et al. ${ }^{22}$, the corresponding increased IOP was $1.496 \mathrm{mmHg}$; in our study, the IOP measured values by Corvis ST increased $1.3 \pm$ $2.4 \mathrm{mmHg}$.

Since BCL wearing increased the apparent CCT values, we may refer to CCT correction method of IOP to minimize the influence of BCL wearing on IOP measurement. Pentacam's five IOP correction formulas were tried and evaluated respectively. We compared the corrected IOP after BCL wearing with the uncorrected IOP before BCL wearing, and found that the difference of Kohlhaas formula was the most significant. Kohlhaas formula was initially developed to amend the IOP measurement after Laser 
Assisted In-situ Keratomi (LASIK) in which a corneal flap is created, and a modified constant of $0.75 \mathrm{mmHg}$ was added ${ }^{12}$. So it might not be suitable to use Kohlhaas formula for the correction of IOP after BCL wearing, which would cause overestimation of IOP measurements. Among the other formulas, the difference of Ehlers formula was the smallest, followed by Orssengo/Pye formula. Therefore, when using a NCT to measure the IOP over BCL wearing, it is recommended to use the Pentacam IOP correction system with Ehlers formula and Orssengo/Pye formula for IOP correction.

Previous studies had shown that the interaction among central thickness, lens power and elastic modulus of corneal contact lenses could better explain the effect of corneal contact lenses wearing on IOP measurements ${ }^{17,23}$. Patel et al. ${ }^{16}$ studied siloxane hydrogel contact lenses with low water content (24\%) and high modulus of elasticity (1.2MPa) and hydrogel contact lenses with high water content (69\%) and low modulus of elasticity $(0.91 \mathrm{MPa})$. They found out that the increase of IOP of low elastic modulus contact lenses was related to the central thickness of corneal contact lenses, and the increase of IOP of high elastic modulus contact lenses was more related to the material. The BCL used in our study was lotrafilcon A silicon hydrogel lens with $24 \%$ water content, which is a high elastic modulus lens. Therefore, in addition to the increase of the thickness of corneal contact lens, the elastic modulus of the lens material may also be a factor affecting the measured value of IOP in our study.

Corvis ST is supposed to biomechanically correct the elastic modulus ${ }^{7}$. An IOP correction formula was developed by Joda et al. ${ }^{7}$ through the analysis of clinical data, which is based on the study of finite element parameters. It can reduce the influence of corneal stiffness and age on IOP measurements, and biomechanically correct the IOP values measured by Corvis ST. In fact, study has found out that the biomechanical correction of Corvis ST measurements is closely related to the CCT, and the BIOP can well eliminate the effect of corneal thickness ${ }^{14}$. In our study, the biomechanically corrected IOP (BIOP) after $\mathrm{BCL}$ wearing was significantly lower than the uncorrected IOP before BCL wearing. We speculated BIOP may overcorrected the IOP of eye with BCL. The BCL wearing may affect the accurate measurement of CCT by Corvis ST, therefore an overcorrection of IOP might happen due to the underlying correction logic of BIOP. In addition, this formula was developed to eliminate the effect of age on the elastic modulus of cornea, but the change of elastic modulus caused by BCL is unrelated to age. Therefore, the BIOP offered by Corvis ST may not be suitable for IOP correction in BCL wearers.

In summary, BCL wearing can overmeasure the IOP, either by NCT or Corvis ST. It was related to the increase of the apparent corneal thickness measurement, as well as the change in the elastic property of cornea-BCL complexus. Certain IOP correction systems have been developed to minimize the effect of $\mathrm{BCL}$ wearing on IOP measurement. Pentacam IOP correction system with the Ehlers formula and Orssengo/Pye formula are recommended to correct the IOP values measured by NCT over BCL. Ehlers formula is also recommended when Corvis ST is used to measure the IOP over BCL.

\section{Declarations}

\section{Ethical approval and informed consent:}


Institutional Review Board approval were obtained from the Human Research Ethics Committee at Wenzhou Medical University. Informed consent was obtained from all subjects. The study was conducted under the ethical standards outlined in the Declaration of Helsinki.

\section{Consent for publication:}

Not applicable.

\section{Availability of data and materials:}

The datasets generated and/or analysed during the current study are not publicly available due to the protection of patients' privacy but are available from the corresponding author on reasonable request.

\section{Competing interest:}

All authors have no competing interests that might be perceived to influence the results and/or discussion reported in this paper.

\section{Funding:}

This study was supported by the Natural Science Foundation Project of Zhejiang Province (LWY20H120001), the General Project of Education Department of Zhejiang Province (Y201738690), and the Medical and Health Project of Major Scientific and Technological Innovation of Wenzhou City (ZY2019012), the Medical Health Science and Technology Project of Zhejiang Provincial Health Commission (2019KY466).

\section{Authors' contribution:}

DC and JQ designed the experiment. XTC and YQ performed the experiments. SXL, ZWC and YQ provided the clinical evaluation. DC, XTC, ZWC and FL analyzed the data. XTC and DC wrote the paper. All authors reviewed the manuscript and gave final approval of the version to be published.

\section{Acknowledgement:}

Not applicable.

\section{References}

1. Sekundo W, Dick HB, Meyer CH: Benefits and side effects of bandage soft contact lens application after LASIK: a prospective randomized study. Ophthalmology 2005, 112:2180-3.

2. Kymionis GD, Liakopoulos DA, Grentzelos MA, Diakonis VF, Klados NE, Tsoulnaras KI, Tsilimbaris MK, Pallikaris IG: Combined topical application of a regenerative agent with a bandage contact lens for the treatment of persistent epithelial defects. Cornea 2014, 33:868-72. 
3. Fraunfelder FW, Cabezas M: Treatment of recurrent corneal erosion by extended-wear bandage contact lens. Cornea 2011, 30:164-6.

4. Chen D, Lian Y, Li J, Ma Y, Shen M, Lu F: Monitor corneal epithelial healing under bandage contact lens using ultrahigh-resolution optical coherence tomography after pterygium surgery. Eye Contact Lens 2014, 40:175-80.

5. H L, Y W, R D, P W, J Z, W Z, L L: Intraocular Pressure Changes and Relationship With Corneal Biomechanics After SMILE and FS-LASIK. Investigative ophthalmology visual science 2016, $57: 4180-6$.

6. J H, J X, A W, Deng SX, Cui X, Yu X, Sun X: A new tonometer-the Corvis ST tonometer: clinical comparison with noncontact and Goldmann applanation tonometers. Investigative ophthalmology \& visual science 2013, 54:659-65.

7. AA J, MM S, D K, A E: Development and validation of a correction equation for Corvis tonometry. Computer methods in biomechanics biomedical engineering online 2016, 19:943-53.

8. R V, A E, CJ R, R A, Kang DSY, Lopes BT, Morenghi E, Azzolini C, Vinciguerra P: Influence of Pachymetry and Intraocular Pressure on Dynamic Corneal Response Parameters in Healthy Patients. J Refract Surg 2016, 32:550-61.

9. N E, T B, S S: Applanation tonometry and central corneal thickness. Acta ophthalmologica 1975, 53:34-43.

10. S S, A C, M M, P KS, J K, D H, D M: Relationship between corneal thickness and measured intraocular pressure in a general ophthalmology clinic. Ophthalmology 1999, 106:2154-60.

11. M K, AG B, E S, A P, J GH, E PL: Effect of central corneal thickness, corneal curvature, and axial length on applanation tonometry. Archives of ophthalmology (Chicago, III: 1960) 2006, 124:471-6.

12. $M$ K, E S, AG B, K P: A correction formula for the real intraocular pressure after LASIK for the correction of myopic astigmatism. Journal of refractive surgery $2006,22: 263-7$.

13. GJ O, DC P: Determination of the true intraocular pressure and modulus of elasticity of the human cornea in vivo. Bulletin of mathematical biology 1999, 61:551-72.

14. F B, Z H, J H, J W, M D, L L, A Y, Q W, A E: Clinical Evaluation of Methods to Correct Intraocular Pressure Measurements by the Goldmann Applanation Tonometer, Ocular Response Analyzer, and Corvis ST Tonometer for the Effects of Corneal Stiffness Parameters. Journal of glaucoma 2016, 25:510-9.

15. CW M: Noncontact tonometry through soft contact lenses. American journal of optometry physiological optics 1986, 63:948-51.

16. S P, G S: Influence of lens material and intraocular pressure on the outcome of non-contact tonometry over soft contact lenses. Cont Lens Anterior Eye 2009, 32:68-72.

17. YC L, JY H, IJ W, FR H, YC H: Intraocular pressure measurement with the noncontact tonometer through soft contact lenses. J Glaucoma 2011, 20:179-82. 
18. Q CYWAZ: The measurement of intraocular pressure by non-contact tonometer through bandage contact lenses. Chinese Journal of Practical Ophthalmology 2015, 33:1240-2.

19. F Z, P C, B D, L L, L Z, G SP: The effect of hydrogel and silicone hydrogel contact lenses on the measurement of intraocular pressure with rebound tonometry. Cont Lens Anterior Eye 2011, 34:2605.

20. KC O: The influence of lens power and center thickness on the intraocular pressure measured through soft lenses: a comparison of two noncontact tonometers. Cont Lens Anterior Eye 2012, 35:118-28.

21. Y ZYZJBALXJ: Effects of central corneal thickness and corneal curvature on measurement of intraocular pressure with Goldmann applanation tonometer and non-contact tonometer. CHINESE JOURNAL OF OPHTHALMOLOGY 2009, 45:713-8.

22. MJ D, ML Z: Human corneal thickness and its impact on intraocular pressure measures: a review and meta-analysis approach. Survey of ophthalmology 2000, 44:367-408.

23. S P, W I: Non-contact tonometry over soft contact lenses: effect of contact lens power on the measurement of intra-ocular pressure. Cont Lens Anterior Eye 2004, 27:33-7.

\section{Figures}

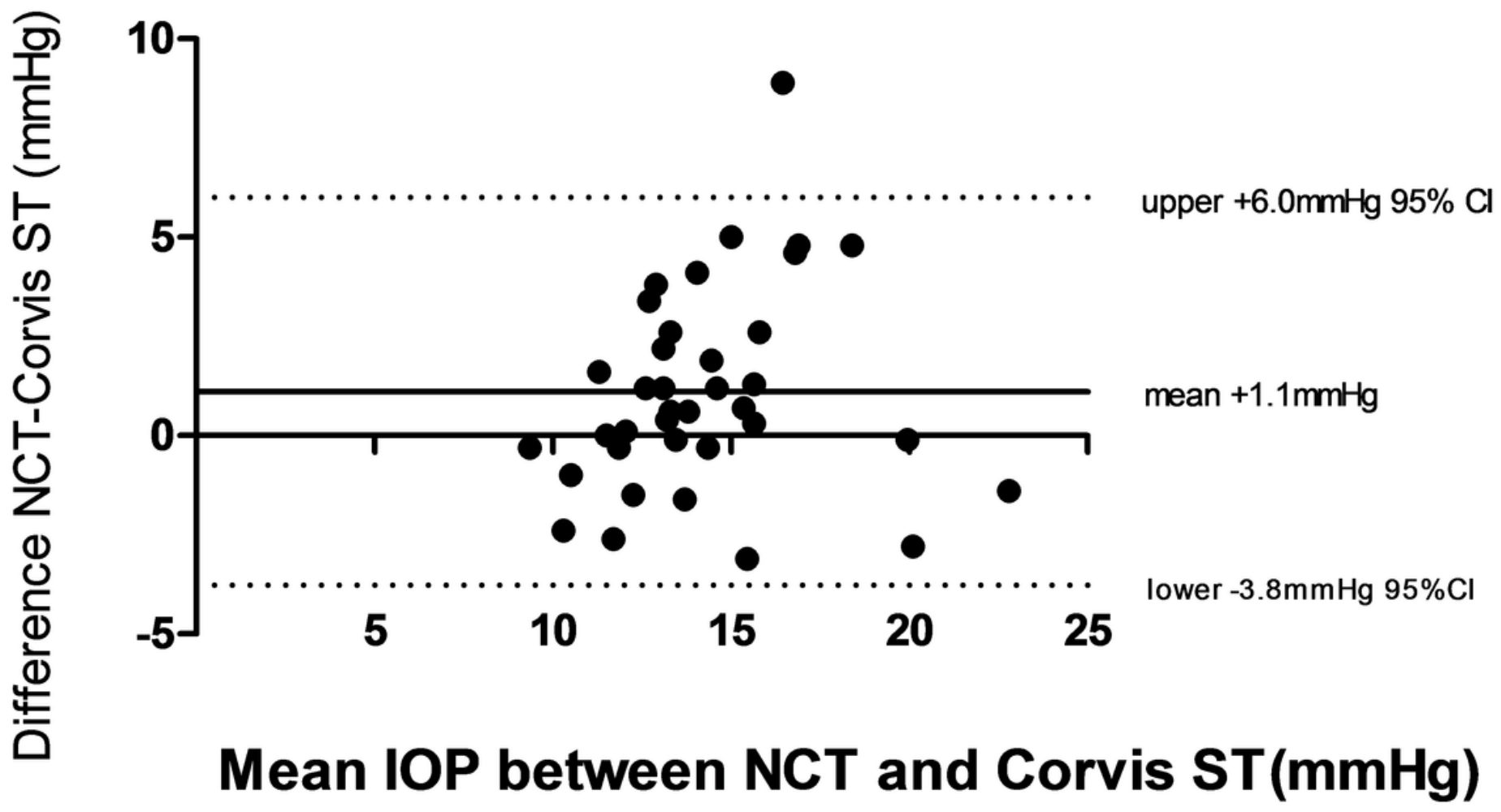

Figure 1 
The Bland-Altman plots for the comparison between the IOP values obtained by non-contact tonometer and Corvis ST before BCL wearing. IOP values from non-contact tonometer was higher than Corvis ST before $\mathrm{BCL}$ wearing $(+1.1 \mathrm{mmHg})$.

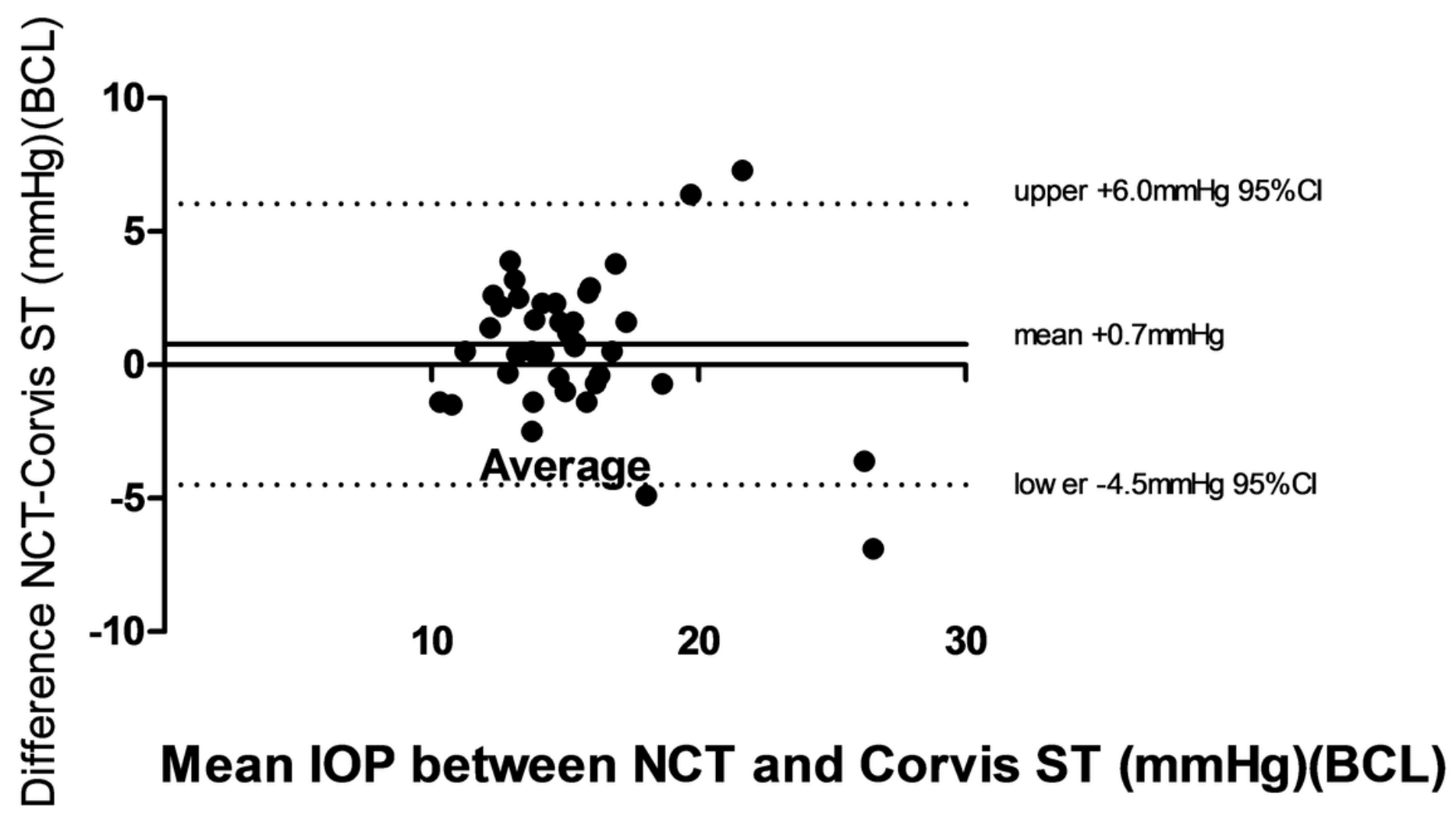

Figure 2

The Bland-Altman plots for the comparison between the IOP values obtained by non-contact tonometer and Corvis ST after BCL wearing. IOP values from non-contact tonometer was higher than Corvis ST after $\mathrm{BCL}$ wearing $(+0.7 \mathrm{mmHg})$. 